\title{
Tip-Specific Functionalization of Gold Nanorods for Plasmonic Biosensing: Effect of Linker Chain
}

\section{Length}

Pedro M. R. Paulo, ${ }^{*}, a$ Peter Zijlstra, ${ }^{b}$ Michel Orrit, ${ }^{c}$ Emilio Garcia-Fernandez, ${ }^{a, \dagger}$ Tamara C. S. Pace, ${ }^{a}$ Ana S. Viana ${ }^{d}$ and Sílvia M. B. Costa ${ }^{a}$

${ }^{a}$ Centro de Química Estrutural, Instituto Superior Técnico, Universidade de Lisboa, Av. Rovisco Pais 1, 1049-001 Lisboa, Portugal.

${ }^{b}$ Molecular Biosensing for Medical Diagnostics, Department of Applied Physics, Eindhoven University of Technology, Eindhoven, The Netherlands.

${ }^{c}$ MoNOS, Huygens Laboratorium, Universiteit Leiden, 2300 RA Leiden, The Netherlands.

${ }^{d}$ Centro de Química e Bioquímica, Faculdade de Ciências da Universidade de Lisboa, Ed. C8, Campo Grande, 1749-016 Lisboa, Portugal.

† Current address: Department of Physical Chemistry, Faculty of Pharmacy, University of Granada, Campus Cartuja, 18071 Granada, Spain

Corresponding Author E-mail: pedro.m.paulo@tecnico.ulisboa.pt 


\begin{abstract}
Gold nanorods are promising platforms for label-free biosensing. We have functionalized gold nanorods with biotin thiol linkers of increasing chain length and evaluated their ability for molecular detection of streptavidin. We have found an unexpected effect for the increase of linker length, as it resulted in a substantial improvement of the plasmon response at surface saturation. The plasmon peak shift increased from $5 \mathrm{~nm}$ to $14 \mathrm{~nm}$, i.e. more than twice the response, between the short and long biotin linker. This effect is only observed for a site selective tip functionalization, whereas for a full biotin coating there is no improvement observed with the linker length. The improved plasmon response for tip functionalization is attributed to a low biotin coverage, but directed to the most sensitive regions, which combined with a longer chain linker reduces steric hindrance for streptavidin binding on the rod's surface. The model sensors were further characterized by measuring their dose-response curves and binding kinetic assays. Simulations of discrete dipole approximation give theoretical plasmon shifts that compare well with the experimental ones for the long linker, but not with those of the short linker, thus suggesting that sterical hindrance affects the latter. Our results highlight the importance of specifically functionalizing the plasmonic hot-spots in nanoparticle sensors with the adequate density of receptors in order to maximize their response.
\end{abstract}

KEYWORDS: Label-free detection; Gold nanorods; Plasmonic sensors; Surface functionalization; Molecular recognition 


\section{Introduction}

Label-free detection of biomolecules plays a key role in the development of affinity-based point-of-care solutions for analysis in areas as diverse as clinical diagnostics or food control. ${ }^{1-3}$ In the process of label-free detection, the target biomolecule when present in the sample will interact at a certain point with a transduction element, either optical, electrical, mechanical or other. Since these interactions usually occur at the surface of the transduction element, the surface chemistry used in the sensor design takes a crucial part in the sensing process. Most important, the functionalization with receptors imparts specificity by only binding the target analyte with high affinity. In this respect, many different types of biomolecular interactions have been explored using receptors based on antibodies, ${ }^{4-7}$ peptides, ${ }^{8}$ aptamers, ${ }^{9,10}$ carbohydrates, ${ }^{11,12}$ or nucleic acids. ${ }^{13,14}$

Colloidal gold particles have interesting optical properties that have been explored in label-free detection schemes. ${ }^{15-18}$ The strong absorption or scattering of light by metal nanoparticles at characteristic wavelengths is due to localized surface plasmon (LSP) modes. The LSP resonance wavelength depends on the plasmon mode and in turn on the particle shape, size and composition. Moreover, it also depends on the local refractive index close to the particle's surface. This feature allows to probe molecular binding events by monitoring the response of the LSP peak wavelength as a function of time. The sensors operating on this principle fall within the general definition of plasmonic sensors.

Particularly, gold nanorods and other elongated particle shapes have been employed for plasmonic sensing because their plasmon resonance is more sensitive to local refractive index than spherical particles. ${ }^{19-25}$ The ultimate goal of single molecule detection has been demonstrated by using gold nanorods for label-free detection of single proteins. ${ }^{26,27}$ The plasmon 
shift induced by molecular binding depends on the refractive index contrast in the overlap integral between the local field and the volume of the molecule. This local field strongly decays away from the particle surface, therefore molecular binding at or near the particle's surface ensures that signal transduction is most effective, however, the sensitivity is usually not homogeneously distributed across the surface. ${ }^{28}$ The plasmon resonance gives rise to intense near fields with a surface distribution that depends on the mode profile, and thus on the particle geometry. In gold nanorods, the largest near-field enhancements are concentrated at the tips. These regions are known as 'hot-spots' for plasmonic sensing, because the sensitivity to local changes in the refractive index is highest there.

Recent reports have shown the site-specific functionalization of nanoparticles with the aim to maximize the sensor sensitivity. ${ }^{27,29-34}$ For example, we have shown that tip specific functionalization of gold nanorods enables the maximization of sensor response for single protein detection by avoiding the binding of the target molecule in regions without sensitivity. ${ }^{27}$ Furthermore, this type of functionalization preserves the quality factor of the plasmonic sensor because it avoids line broadening due to chemical interface damping, in contrast to a full surface coverage with thiolated receptors. ${ }^{32}$ Other studies on the selective functionalization of plasmonic hot-spots, such as edge or vertex sites in gold nanoplates or gaps in dimers of gold disks, have also shown that optimum sensitivity can be attained by functionalizing regions that exhibit a high local field. ${ }^{29-31}$ Those studies have addressed the effect of linker length, and found the expected dependence, namely, that the plasmon sensitivity decreases upon increasing the linker chain length.

In this work, we report on the effect of the linker length in the response of a tip-functionalized nanorod sensor. To this end gold nanorods were immobilized on a glass substrate to allow for 
transmission probing of plasmon shifts. The nanorods were tip-functionalized on the glass surface using a protocol that has previously proved to be site-selective. ${ }^{27,32}$ The functionalization was done with three biotin linkers of increasing chain length (Fig. 1A and Table S1 in the SI). The performance of the nanorod sensor was evaluated by measuring the LSP peak shift upon streptavidin binding (Fig. 1B). We find a strong dependence of the plasmon shift on linker length where, surprisingly, the longer chain linkers give larger plasmon shifts, in contrast to the expected trend where longer linkers result in decreased signal. On the other hand, the fully functionalized particles do not show almost any increase of the plasmon shift with the linker length, which we attribute to the high density of biotin receptors in a full surface functionalization that hinders streptavidin binding and imposes a larger distance to the particle's surface, thus reducing the sensor response. Tip-specific functionalization seems to result in a lower density of biotin receptors at the nanorod's hot-spots, that combined with longer and flexible linkers, favors streptavidin binding and maximizes the sensor response.

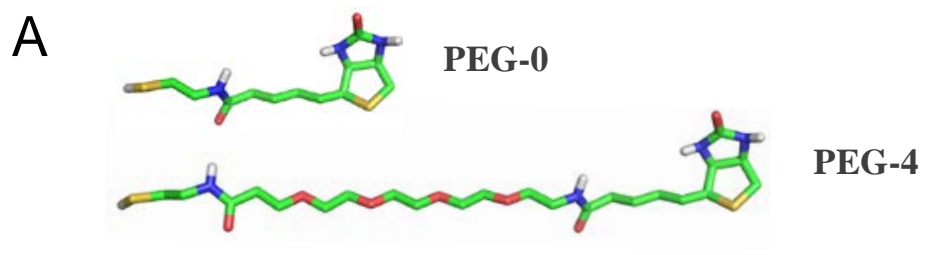

PEG-12
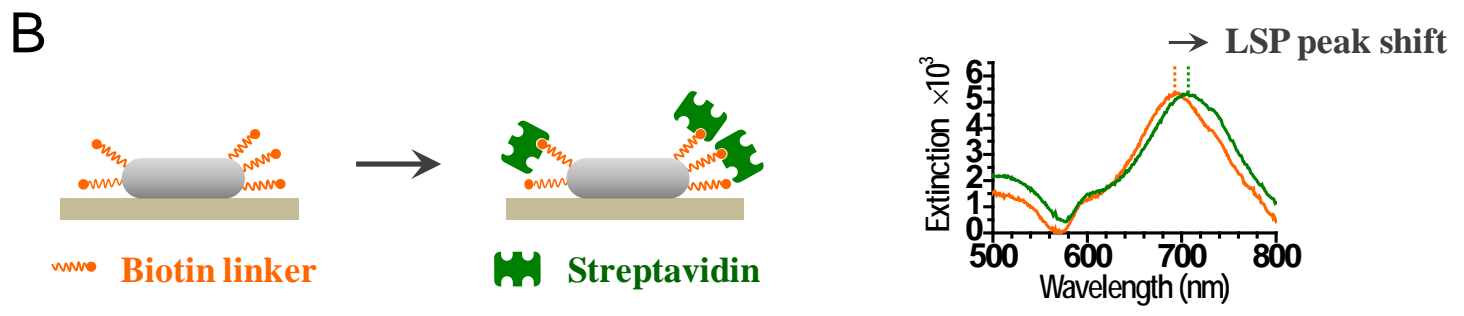

Figure 1. A) Chemical structures of the biotin thiol linkers: PEG-0; PEG-4; and PEG-12. B) Scheme of the principle of plasmonic sensing using the peak shift of the longitudinal surface 
plasmon (LSP) of gold nanorods tip-functionalized with biotin (orange) for specific binding of streptavidin (green).

\section{Experimental Section}

\section{Materials and Instruments}

Gold nanorods coated with CTAB and approximate size of $10 \mathrm{~nm} \times 30 \mathrm{~nm}$ were acquired from Nanopartz Inc. as aqueous suspensions with an optical density of 1 or 100 (product \# A12-10700 or A12C-10-700). Precursors for the biotin thiol linkers of commercial names: EZ-Link ${ }^{\mathrm{TM}}$ NHS- Biotin; EZ-Link ${ }^{\mathrm{TM}}$ NHS-PEG4-Biotin; and EZ-Link ${ }^{\mathrm{TM}}$ NHS-PEG12-Biotin, were acquired from Fisher Scientific and used as received. Cysteamine was from Sigma with a purity above 98\%. (3-Mercaptopropyl)trimethoxysilane was from Aldrich with a purity of 95\%. Hexadecyltrimethylammonium bromide or CTAB was from Sigma, BioXtra grade. Surface coating reagent methyl-PEG4-thiol was from Fisher Scientific and used as received. Streptavidin from Streptomyces avidinii was acquired from Sigma, already affinity purified and lyophilized from $10 \mathrm{mM}$ potassium phosphate. Biotin was from Sigma-Aldrich with purity above 99\%. Phosphate buffer saline (PBS) buffer was acquired as tablets from Sigma, and dissolved in MilliQ (Merck Millipore) deionized water. Ethanol absolute for UV, IR, HPLC was from Panreac, and toluene for spectroscopy, Uvasol grade, was from Merck. Glass slides of rectangular shape with thicknesses \# 1 and 1.5 were acquired from Menzel-Gläser or Deltalab.

Extinction spectra were measured in an UV/Vis spectrophotometer from PerkinElmer, model Lambda 35. Glass surfaces were cleaned using an UV/Ozone chamber model PSD-UV3 from Novascan. AFM measurements were performed in tapping mode using a Nanoscope IIIa multimode microscope from Digital Instruments, Veeco. AFM imaging was carried under 
ambient conditions using silicon tips, TESP-SS from Bruker AFM Probes, with a resonance frequency of ca. $320 \mathrm{kHz}$, at a scan rate of $1.0-1.5 \mathrm{~Hz}$.

Immobilization of gold nanorods on glass slides

Glass coverslips $24 \times 60 \mathrm{~mm}$ of thickness \# 1.5 were thoroughly cleaned by performing a UV/ozone treatment for 90 min followed by immersion in $\mathrm{HCl}(1 \mathrm{mM})$ for $1 \mathrm{hr}$, then rinsing copiously with water and, finally, by sonicating in water and methanol for 20 mins with intermediate steps of $\mathrm{N}_{2}$ blow drying. The clean slides were silanized by immersion in (3Mercaptopropyl)trimethoxysilane, 5\% (v/v) in ethanol, then rinsed copiously with ethanol and sonicated during 20 min in methanol. The silane coating creates a glass surface functionalized with thiol groups that was used to covalently attach the gold nanorods for the subsequent surface chemistry.

Gold nanorods were centrifuged and the supernatant was replaced with an aqueous solution of CTAB $(50 \mu \mathrm{M})$ for at least three times, in order to wash down CTAB concentration and facilitate surface adhesion. The washed suspension of particles was dispensed on the silanized glass surfaces, over an area of approximately $1 \mathrm{~cm}^{2}$, by leaving a $300 \mu \mathrm{L}$ drop in contact with the glass for 20 min then rinsing copiously with water and blow drying with $\mathrm{N}_{2}$. This procedure was repeated 3 to 5 times until the particle density on the glass surface reached an O.D. of 0.003 to 0.010 .

Tip-specific functionalization of gold nanorods

The biotin thiol linkers were prepared by a coupling reaction between the EZ-Link ${ }^{\mathrm{TM}}$ precursor (10 mM) with cysteamine $(1 \mathrm{mM})$ in PBS buffer. The reaction was allowed to proceed 
for 30 min before the mixture was used without purification in the following functionalization steps. The biotin thiol linkers were named after the length of the oligoethylene glycol spacer in the precursor: PEG-0, PEG-4 and PEG-12, respectively for NHS-Biotin; NHS-PEG4-Biotin; and NHS-PEG12-Biotin.

The glass slides containing gold nanorods were gently cleaned by $10 \mathrm{~min}$ of UV/ozone treatment, then rinsed with water and blow-dried with $\mathrm{N}_{2}$. The first step of tip-specific functionalization was the incubation of the area where rods were immobilized with a CTAB solution (ca. $1 \mathrm{mM}$ ) for about 30 mins. Then, the CTAB solution was replaced with a solution of biotin thiol linker (1, 10 or $100 \mu \mathrm{M}$ ) and CTAB (ca. $1 \mathrm{mM}$ ). The CTAB molecules present during the functionalization step create a bilayer coating over the nanorods that is more compact over the side of the rods, thereby favoring the thiol attachment at the tips. The thiol attachment reaction was allowed to proceed for $90 \mathrm{~min}$. Finally, the surface was thoroughly rinsed with water and left overnight immersed in PBS buffer to remove any reactant residues. The thorough removal of CTAB surfactant is important to avoid any interference during the sensing assays.

Alternatively, to achieve a full coating with the biotin thiol linkers, the CTAB detergent was not added to the thiol solution. The glass surfaces with the gold nanorods were exposed to an aqueous solution of the biotin thiol linker $(1 \mathrm{mM})$ for $90 \mathrm{~min}$, and after the surface was cleaned with the same protocol as before.

\section{Streptavidin sensing with functionalized gold nanorods}

The glass slides containing the biotin functionalized gold nanorods were assembled into a liquid cell by attaching over each one a clean glass slide of thickness \# 1using a melted thin frame of Parafilm M. The liquid cells are about $1 \mathrm{~mm}$ thick and hold approximately $500 \mu \mathrm{L}$ of 
solution. Before the sensing experiments, each liquid cell was left overnight in PBS buffer to remove any additional residues. A stock solution of streptavidin was prepared by dissolving 1 mg of protein in $1 \mathrm{~mL}$ of PBS. This solution was diluted in PBS buffer to obtain the final concentration of streptavidin. The extinction spectra of the liquid cell filled with PBS was recorded to obtain the initial peak position of the longitudinal surface plasmon (LSP). Then, the PBS buffer was replaced with a streptavidin solution, and consecutive spectra were recorded each minute, over a wavelength span of $100 \mathrm{~nm}$ around the peak position at a speed of 120 $\mathrm{nm} / \mathrm{min}$. After each kinetic run, that typically lasts 90 or $120 \mathrm{~min}$, the streptavidin solution may be replaced with another of higher concentration to perform another kinetic run. This procedure was used, for instance, to test the full output response of each surface by using a streptavidin concentration high enough to saturate all biotin receptors. The gold nanorods are firmly attached to the glass surface which precludes any eventual interference effect from particle aggregation on the plasmon peak shift.

The kinetic traces are built from a time series of extinction spectra. Each peak was fitted with a Gaussian function and the maximum wavelength retrieved from the fit was represented as a function of time. The kinetic traces were fitted with a single exponential or a stretched exponential function, and the quality of the fits was evaluated from the residuals plot. The plasmon (LSP) peak shift was evaluated as the difference between the value of the fitted exponential function at the end of the trace and the initial peak position.

\section{DDA simulations}

We have used the discrete dipole approximation (DDA) method to evaluate the extinction cross-section and the near-field enhancement of gold nanorods. ${ }^{35}$ The particle geometry used was 
that of a spherically capped cylinder discretized as an array of point dipoles centered in cubic volume elements with size of $0.25 \mathrm{~nm}$. The dielectric function of gold, as reported by Johnson and Christy, was used in these calculations. ${ }^{36}$ It was scaled by dividing with the dielectric constant of the solvent to simulate the surrounding environment. The details on the coated particle models used to simulate the functionalization with the linker layer and the binding of the target layer are given in the supporting information. The near-field intensity maps were calculated using the subroutine implemented in the ADDA software package. ${ }^{37}$

\section{Results and Discussion}

Gold nanorods that have been functionalized with biotin thiol linkers respond to nanomolar concentrations of streptavidin by displaying a red-shift of their longitudinal LSP peak (Fig. 2A). Time traces of the peak position show that after several minutes the peak shift slows down and eventually it stabilizes suggesting that a dynamic equilibrium between protein association and dissociation has been established (Fig. 2B). The peak shift stabilizes at increasing values when protein concentration is increased up to hundreds of nanomolar. In this range, the peak shift for biotin-functionalized nanorods reaches several nm. In comparison, bare nanorods show only about $1.5 \mathrm{~nm}$ of peak shift in the same concentration range (grey symbols). The control experiments show that the response measured in biotin-functionalized nanorods is from specific adsorption mediated by the strong streptavidin-biotin pair interactions, while in nonfuncionalized nanorods only a marginal response is observed due to non-specific adsorption of protein at the particle's surface (Figs. S1 and S2 in the SI). 

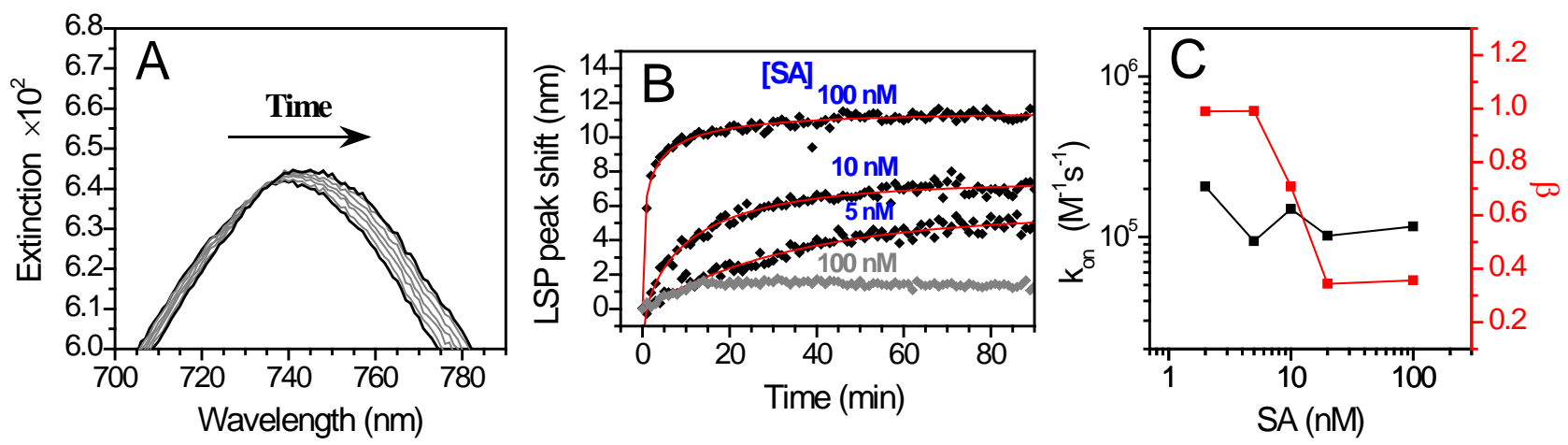

Figure 2. (A) Plasmon (LSP) peak shift in the extinction spectrum of gold nanorods tipfunctionalized with PEG-12 biotin thiol linker in response to streptavidin binding. (B) Kinetic traces showing the change in the LSP peak position over time for several concentrations of streptavidin (SA), as indicated in the figure. The grey symbols show the kinetic trace of a control experiment performed with bare (non-functionalized) gold nanorods. The red curves show the fits performed with a stretched exponential. (C) Values of the binding rate constant, $k_{\mathrm{on}}$, and exponent $\beta$ obtained from fitting kinetic traces.

The kinetic traces are approximately exponential at low protein concentration, but deviate considerably from first order kinetics at higher concentrations. The fittings using a stretched exponential yielded values of binding rate constant, $k_{\text {on, }}$ around $10^{4}-10^{5} \mathrm{M}^{-1} \mathrm{~s}^{-1}$ with an exponent $\beta$ varying from 1 to 0.3 as protein concentration increases (Fig. 2C). The non-exponential behavior resulting in $\beta$ values well below unit is typically associated with heterogeneity in reaction kinetics at surfaces, ${ }^{38,39}$ e.g. due to steric hindrance or surface charge effects. In these conditions, the Langmuir adsorption model with a single adsorption rate constant might not suffice to describe the heterogeneous adsorption process. Moreover, a transition between diffusion and reaction-limited regimes is another probable source of complexity for the binding 
kinetics. At low protein concentrations, the adsorption rates are most likely diffusion limited, similar to other studies on the adsorption of streptavidin onto SAMs of biotin receptors. ${ }^{40-44}$ The weak variation in the initial adsorption rates for the different linkers and conditions used in this work seems to support this hypothesis. As protein concentration is increased and mass transport becomes faster, it occurs a transition to a partially reaction-limited regime that shows up in the adsorption kinetics (see SI for further details). At higher surface coverage, protein interactions due to crowding on the surface of the particle again lower the adsorption rates. The consequence for the binding kinetics would be equivalent to a broad distribution of adsorption rate constants, as opposed to a Langmuir type of adsorption with a single rate constant. This argument holds for both non-specific adsorption and for specific streptavidin-biotin binding. In the latter case, the availability of biotin receptors on the nanorod's surface can be hindered by interactions with neighboring chains or by nearby streptavidin already bound, and further contribute to the emergence of heterogeneity in adsorption kinetics.

The effect of the thiol linker length in the specific response of biotin-functionalized gold nanorods to streptavidin adsorption was characterized by their dose-response curves (Fig. 3). The dynamic range for streptavidin detection extends from ten to about one hundred nanomolar of streptavidin. At protein concentrations below $10 \mathrm{nM}$, the measured peak shifts are below the threshold of $1.5 \mathrm{~nm}$ (dashed line), which is the non-specific response measured in bare nanorods at full surface coverage. Above micromolar concentrations, the limit of receptor saturation is reached and the LSP peak shift is saturated. 

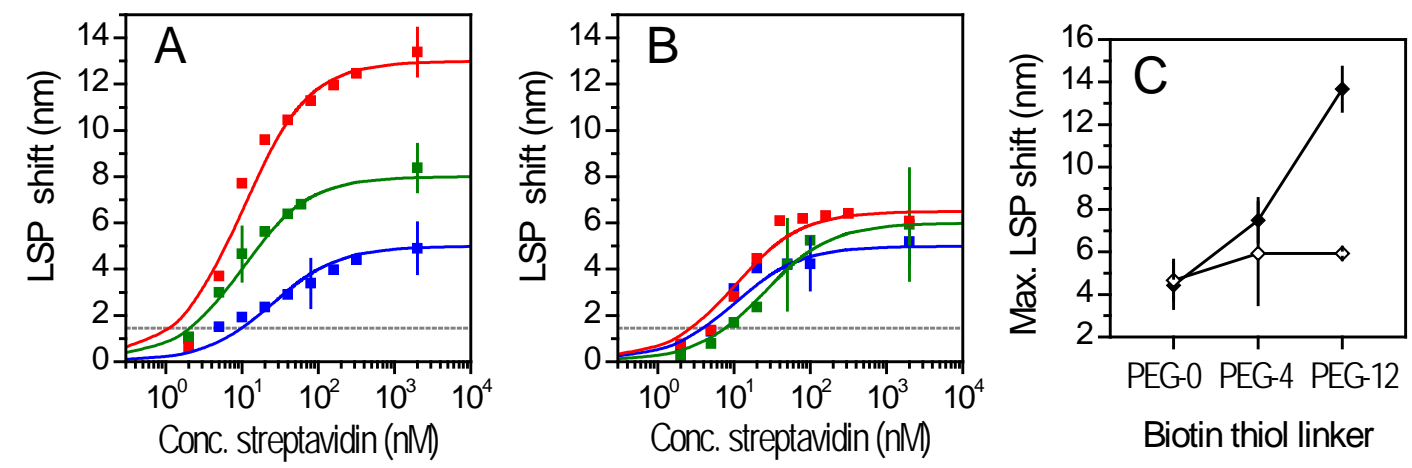

Figure 3. Dose-response curves for plasmonic sensing of streptavidin with gold nanorods: (A) tip-functionalized; (B) fully coated with biotin thiol linkers: PEG-0 (blue); PEG-4 (green); and PEG-12 (red). The error bars represent the standard deviation for at least three independent measurements of the same streptavidin concentration. The curves shown were adjusted with a Langmuir adsorption type of model. The horizontal dashed line represents the full response for non-specific adsorption measured for bare gold nanorods. (C) Plasmon peak shift measured at surface saturation (full response) for streptavidin sensing with gold nanorods: tip-functionalized (closed symbols) or fully coated (open symbols) with different biotin thiol linkers.

The dose-response curves were fitted with a 1:1 binding model based on a Langmuir type of adsorption used here to describe the specific streptavidin-biotin interaction on the nanorod's surface. As previously noted, this approach might be over-simplistic to describe these systems. Nevertheless, it allows to extract an apparent association constant, $K_{\mathrm{A}}$, for the streptavidin-biotin pair interaction. The values obtained fall in the range of $10^{7}-10^{8} \mathrm{M}^{-1}$ (Table S2 in the SI). These values of binding affinity are much lower than the well-known value of $10^{13} \mathrm{M}^{-1}$ that makes streptavidin-biotin interaction one of the strongest biomolecular interactions. ${ }^{45,46}$ The values of $K_{\mathrm{A}}$ from our assays are probably affected by the reduced response of our sensor at low protein 
concentrations due to mass transport limitations. As a consequence, the dose-response curves do not reflect the actual $K_{\mathrm{A}}$ from the streptavidin-biotin pair association, but rather the protein concentration at which diffusion becomes efficient to deliver it to the surface on the time interval of our assays. Our values of $K_{\mathrm{A}}$ compare well with several other examples of binding affinity assays of streptavidin-biotin pair measured with plasmonic sensors. ${ }^{20,21,47-52}$ Nevertheless, there is a wide range of $K_{\mathrm{A}}$ values reported in the literature, which most likely are related to the specific details of each binding assay. For instance, large values of $K_{\mathrm{A}}$ have been reported for long incubation times at low protein concentrations, or incubation under flow conditions, to overcome mass transport limitations. . $33,54^{2}$

The full response of our plasmonic sensors at surface saturation changes with the biotin linker chain length for the tip-functionalized gold nanorods. Counterintuitively, the longer PEG-12 linker shows the largest peak shift of ca. $14 \mathrm{~nm}$, while the shorter PEG-0 linker gives a full peak shift of only $5 \mathrm{~nm}$. The initial guess was that the shorter linker would give the largest response because it would bind streptavidin closer to the nanorod's surface in a region of larger near field enhancement and, thus, of larger refractive index sensitivity. However, the sparse density of biotin linkers in tip functionalization probably still allows for streptavidin to approach the rod's surface, even if bound to long linkers, because of their flexible chain. On the other hand, the linker effect just described is not observed for a full functionalization, in which case the plasmon response at surface saturation is around $5 \pm 1 \mathrm{~nm}$, and it practically does not change for the different chain lengths investigated. The full functionalization gives always lower sensor responses at surface saturation than tip functionalization with the same thiol linker, except for the short PEG-0 linker that has practically the same the response in tip and full functionalization. The PEG-0 linker has a length comparable to the depth of the biotin binding pocket of 
streptavidin, which hinders protein interactions with biotin on the rod's surface. The longer linkers relieve some of the steric hindrance in the streptavidin-biotin interactions and probably favor the binding of more proteins, thus giving larger sensor responses in tip functionalization; whereas in full functionalization, the molecular packing of linker chains prevents the protein from approach the rod's surface, where plasmon sensitivity is larger.

We have also investigated how the density of biotin receptors affects the full peak shift at surface saturation by changing the concentration of the PEG-12 linker in the functionalization step of the tip-specific procedure (Fig. 4). At low concentration, the full sensor response is smaller due to a low density of biotin receptors. It reaches a maximum for an intermediate concentration, before it falls off at the higher concentration used. The optimum concentration of PEG-12 linker in the functionalization step to obtain a maximum sensor response is attributed to a compromise between the number of biotin receptors per rod and the emergence of steric hindrance at high biotin densities. In line with our results, others have reported that steric hindrance on plane substrates can significantly decrease the saturation coverage of streptavidin in SAMs of biotin by 1.5 to 4 -fold. ${ }^{42}$

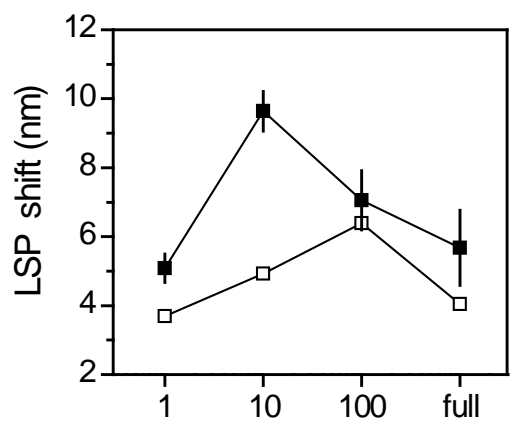

Linker conc. at functionalization $\left(10^{-6} \mathrm{M}\right)$ 
Figure 4. Plasmon peak shift for streptavidin sensing with gold nanorods functionalized with PEG-12 (closed squares) and PEG-0 (open squares) as a function of the linker concentration used at the functionalization step of 1 to $100 \mu \mathrm{M}$ and also for a full functionalization.

The short PEG-0 linker also shows a non-monotonic variation of sensor response as the linker concentration is increased, although in this case the change in trend is observed at a concentration of $100 \mu \mathrm{M}$. We cannot exclude that the larger plasmon response may occur between this concentration and full functionalization, which would suggest that the biotin density for PEG-0 linker was still increasing. While comparing linkers of different length, it should be considered that the number of biotin receptors on the rod's surface may change for linkers of different chain length. For instance, it was found evidence that longer linker chains give lower grafting densities by monitoring the plasmon linewidth of individual nanorods. ${ }^{32}$ The plasmon linewidth is broadened by the number of thiols on the surface through a chemical interface damping mechanism. The functionalization of gold nanorods with long thiolated polyethylene glycol chains gives less line broadening compared to a short chain, which suggests a lower number of attached thiols. However, a lower number of thiols, and thus of biotin receptors on the rods' surface, does not necessarily affect the number of attached streptavidin molecules at surface saturation, if the grafting density corresponds to at least one receptor per surface area occupied by a single protein. A lower grafting density can even be beneficial, if it separates biotin receptors farther apart on the rod's surface, thereby decreasing the steric hindrance due to interaction between binding sites. Our results seem to support this hypothesis as the longer linker gives a larger sensing response in a tip functionalization and practically the same response in a full coating functionalization. 
In order to evaluate molecular crowding of streptavidin binding on the surface of gold nanorods, we have used AFM to obtain topographic information on the gold nanorods functionalized with PEG-12 linker. We have selected this biotin linker because it gives the larger difference in the plasmon peak shift for tip-selective vs. full coating. The fully-functionalized gold nanorods after saturation with streptavidin show an increase of height by ca. $5 \mathrm{~nm}$ which matches well the size of streptavidin (Fig. S11 in the SI). In contrast, the tip-functionalized gold nanorods after saturation with streptavidin show no increase in their height, which suggests that in this case there is much less protein on the side of the particles than in fully-functionalized nanorods.

To understand the effect of the distance of the biotin receptors to the gold surface on the plasmon shift, we have employed numerical simulations of a coated gold nanorod in the discrete dipole approximation (Fig. 5). The thickness of the linker layer was matched to that of PEG-12 linker at two limiting cases: that of a freely rotating chain $($ ca. $1.8 \mathrm{~nm})$ or the fully extended chain (ca. $5.2 \mathrm{~nm}$ ). The peak shifts calculated for the first case reach almost $14 \mathrm{~nm}$ for a target layer having the thickness of a full monolayer of streptavidin (closed squares, Fig. 5C). This value compares well to the experimental peak shift obtained at surface saturation for tip functionalization with PEG-12 linker. This implies that the linkers behave like a freely rotating chain in the case of tip-functionalized nanorods. 

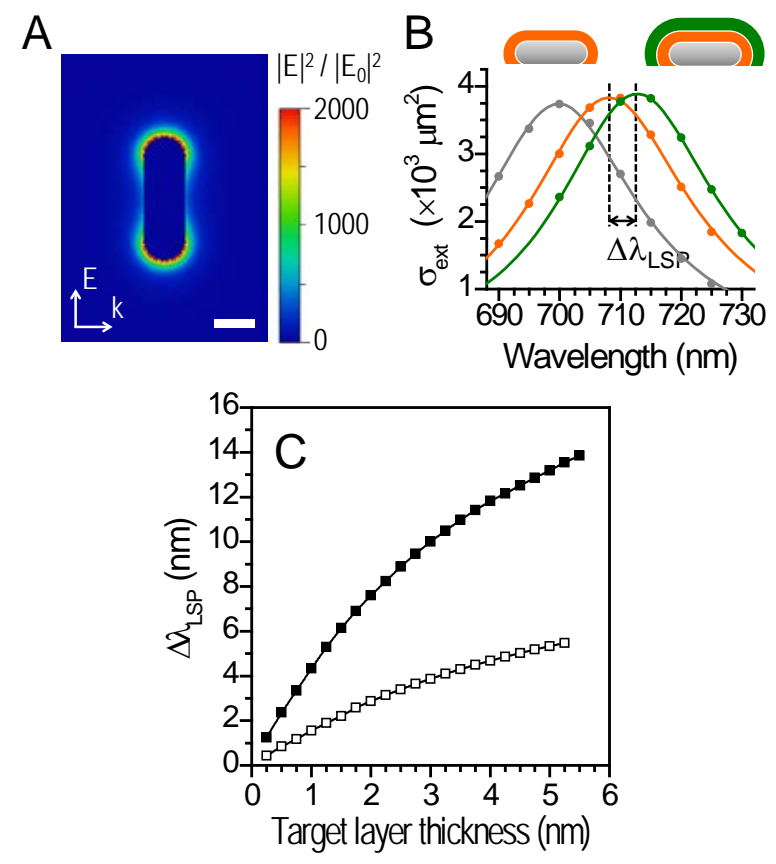

Figure 5. (A) Near field map obtained from DDA simulations of a gold nanorod of $10 \mathrm{~nm} \times 30$ $\mathrm{nm}$ at the longitudinal plasmon resonance wavelength. B) Extinction cross-sections calculated for a gold nanorod: bare (grey); fully coated with a layer to simulate the biotin linker (orange); and, with another layer to simulate the streptavidin target (green) - see inset. The plasmon peak shift $\left(\Delta \lambda_{\text {LSP }}\right)$ is the difference between the maximum wavelengths of the last two situations. (C) Calculated peak shifts for the model of coated gold nanorod with varying target layer thickness up to $5.5 \mathrm{~nm}$ to simulate a single layer of streptavidin. The thickness of the linker layer was matched to that of PEG-12 linker at two limiting cases: a freely rotating chain (closed squares) or a fully extended chain (open squares).

On the other hand, the measured peak shits for fully functionalized particles are in good agreement with a model based on a fully extended linker chain with a biotin group ca. $5 \mathrm{~nm}$ away from the surface (open squares, Fig. 5C). The reduced sensor response for a larger distance 
of target binding matches the maximum peak shift observed for full functionalization with PEG12 linker. A similar argument, based only on the target binding distance, does not explain the lower sensor responses measured for the shorter PEG-0 linker. The calculated plasmon shift for a full target layer would be $11 \mathrm{~nm}$, and the experimental shift of $5 \mathrm{~nm}$ is matched in the calculations by a layer with approximately half the size of a full layer. In the case of biotin PEG0 linker, the surface coverage at saturation is probably hindered by unfavorable interactions between binding sites and the surface due to the short linker length, as previously mentioned. This type of effect could prevent the formation of a compact layer of target protein even at surface saturation.

In order to compare the performance of our nanorod biosensors with other examples reported in the literature, we have determined the refractive index sensitivity and calculated some figuresof-merit. The refractive index sensitivity, $S=d \lambda_{\mathrm{LSP}} / d \eta$, and the figure-of-merit, FOM $=S / \Delta \lambda$, for our gold nanorod surfaces were found to be $239 \mathrm{~nm} / \mathrm{RIU}$ and 0.9 , respectively (see further details in SI). These values are comparable to those reported by Mayer et al. for a gold nanorod based sensor. ${ }^{55}$ The figure-of-merit of our sensor surfaces is below the optimum value of $11 \mathrm{for}$ a gold nanorod of aspect ratio of 4 , as proposed in the literature. ${ }^{19}$ However, our sensing assays were performed in ensemble conditions, and thus the plasmon band linewidths are much larger than those of single nanoparticles used in Ref. 8, which decreases the quality factor of the sensor. Finally, we have also used a definition of a figure-of-merit $\left(\mathrm{FOM}_{\mathrm{mol}}\right)$ based on the minimum number of detectable molecules $(M D L)$. This number can be found using Eq. S4 in the SI from a combination of experimental and estimated parameters. We have calculated the $M D L$ of 3.3 molecules and the $\mathrm{FOM}_{\text {mol }}$ of 9 for our nanorod sensors. This latter is close to the value of 18 
predicted as optimum $\mathrm{FOM}_{\mathrm{mol}}$ for gold nanorods of size $30 \mathrm{~nm} \times 60 \mathrm{~nm}$ also used in molecular detection of streptavidin. ${ }^{56}$

\section{Conclusions}

The performance of label-free sensors strongly relies on the molecular interactions between the target analyte and specific receptors functionalized at the sensing element. This feature is well illustrated by our results on gold nanorods functionalized with biotin receptors for streptavidin sensing. The combination of a sparse biotin functionalization, but directed toward the most sensitive regions of the rod's surface, and of a long chain linker, resulted in an improvement by more than two-fold in the full plasmonic response of gold nanorods that were tip-functionalized with the PEG-12 linker. The expected chain length effect would be that an increase in the linker length leads to a decrease of the plasmon shift for a given protein coverage. However, we found the opposite trend in tip-specific functionalized nanorods. This counterintuitive effect is attributed to the sparse tip-functionalization that combined with longer linkers relieve some of the steric hindrance present in target-receptor molecular interactions at the rod's surface. As a consequence, tip-functionalization with longer linkers seems to allow for a larger number of proteins to bind resulting in a larger signal and enhanced sensor performance. Further studies using single particle or single molecule detection will contribute to elucidate the source of heterogeneity in molecular interactions at surfaces used for plasmonic sensing.

\section{ASSOCIATED CONTENT}

\section{Supporting Information.}


Estimates of linker lengths; Control experiments: blanks and specificity; Kinetics of streptavidin-biotin binding; Refractive Index sensitivity and Figures-of-Merit; Details on the DDA simulations; Height profiles from AFM images.

\section{AUTHOR INFORMATION}

\section{Corresponding Author}

*E-mail: pedro.m.paulo@tecnico.ulisboa.pt.

\section{Notes}

The authors declare no competing financial interest.

\section{ACKNOWLEDGMENTS}

Authors gratefully acknowledge financial support from Fundação para a Ciência e a Tecnologia, FCT (Pest-OE/QUI/UI0100/2013/2014 and PTDC/CTM-NAN/2700/2012). PMRP acknowledges a Post-Doc grant from FCT (SFRH/BPD/111906/2015). PZ acknowledges financial support from The Netherlands Organisation for Scientific Research (VIDI grant). MO acknowledges support from the Dutch Science Agency NWO. ASV acknowledges PEst 20152020-UID/Multi/00612/2013 and IF/00808/2013 (POPH, UE-FSE). PMRP would like to thank A. Pezzarossa for helpful discussions. 


\section{REFERENCES}

1. Hunta, H. K.; Armani, A. M. Label-free biological and chemical sensors. Nanoscale 2010, 2, 1544-1559.

2. Estevez, M.-C.; Alvarez, M.; Lechuga, L. M. Integrated optical devices for lab-on-achip biosensing applications. Laser Photonics Rev. 2012, 6, 463-487.

3. Brolo, A. G. Plasmonics for future biosensors. Nat. Photonics 2012, 6, 709-713.

4. Hall, W. P.; Ngatia, S. N.; Van Duyne R. P. LSPR Biosensor Signal Enhancement Using Nanoparticle-Antibody Conjugates. J. Phys. Chem. C 2011, 115, 1410-1414.

5. Tang, L.; Casas, J. Quantification of cardiac biomarkers using label-free and multiplexed gold nanorod bioprobes for myocardial infarction diagnosis. Biosens. Bioelectron. 2014, 61, 70-75.

6. Tian, L.; Morrissey, J. J.; Kattumenu, R.; Gandra, N.; Kharasch, E. D.; Singamaneni, S. Bioplasmonic Paper as a Platform for Detection of Kidney Cancer Biomarkers. Anal. Chem. 2012, 84, 9928-9934.

7. Chen, P.; Chung, M. T.; McHugh, W.; Nidetz, R.; Li, Y.; Fu, J.; Cornell, T. T.; Shanley, T. P.; Kurabayashi, K. Multiplex Serum Cytokine Immunoassay Using Nanoplasmonic Biosensor Microarrays. ACS Nano 2015, 9, 4173-4181.

8. Tadepalli, S.; Kuang, Z. Jiang, Q. Liu, K.-K.; Fisher, M. A.; Morrissey, J. J.; Kharasch, E. D.; Slocik, J. M.; Naik, R. R.; Singamaneni, S. Peptide Functionalized Gold 
Nanorods for the Sensitive Detection of a Cardiac Biomarker Using Plasmonic Paper Devices. Sci. Rep. 2015, 5, 16206-11.

9. Guo, L.; Zhou, X.; Kim, D.-H. Facile fabrication of distance-tunable Au-nanorod chips for single-nanoparticle plasmonic biosensors. Biosens. Bioelectron. 2011, 26, 22462251.

10. Guo, L.; Kim, D.-H. LSPR biomolecular assay with high sensitivity induced by aptamer-antigen-antibody sandwich complex. Biosens. Bioelectron. 2012, 31, 567570.

11. Yonzon, C. R.; Jeoung, E.; Zou, S.; Schatz, G. C.; Mrksich, M.; Van Duyne R. P. A Comparative Analysis of Localized and Propagating Surface Plasmon Resonance Sensors: The Binding of Concanavalin A to a Monosaccharide Functionalized SelfAssembled Monolayer. J. Am. Chem. Soc. 2004, 126, 12669-12676.

12. Frolov, L.; Dix, A.; Tor, Y.; Tesler, A. B.; Chaikin, Y.; Vaskevich, A.; Rubinstein, I. Direct Observation of Aminoglycoside-RNA Binding by Localized Surface Plasmon Resonance Spectroscopy. Anal. Chem. 2013, 85, 2200-2207.

13. Spadavecchia, J.; Barras, A.; Lyskawa, J.; Woisel, P.; Laure, W.; Pradier, C.-M.; Boukherroub, R.; Szunerits S. Approach for Plasmonic Based DNA Sensing: Amplification of the Wavelength Shift and Simultaneous Detection of the Plasmon Modes of Gold Nanostructures. Anal. Chem. 2013, 85, 3288-3296. 
14. Joshi, G. K.; Deitz-McElyea, S.; Johnson, M.; Mali, S.; Korc, M.; Sardar, R. Highly Specific Plasmonic Biosensors for Ultrasensitive MicroRNA Detection in Plasma from Pancreatic Cancer Patients. Nano Lett. 2014, 14, 6955-6963.

15. Szunerits, S.; Boukherroub, R. Sensing using localised surface plasmon resonance sensors. Chem. Commun. 2012, 48, 8999-9010.

16. Couture, M.; Zhaowa, S. S.; Masson J.-F. Modern surface plasmon resonance for bioanalytics and biophysics. Phys. Chem. Chem. Phys. 2013, 15, 11190-11216.

17. Mayer, K. M.; Hafner, J. H. Localized surface plasmon resonance sensors. Chem. Rev. 2011, 111, 3828-3857.

18. Saha, K.; Agasti, S. S.; Kim, C.; Li, X.; Rotello, V. M. Gold Nanoparticles in Chemical and Biological Sensing. Chem. Rev. 2012, 112, 2739-2779.

19. Becker J.; Trügler, A.; Jakab, A.; Hohenester, U.; Sönnichsen C. The Optimal Aspect Ratio of Gold Nanorods for Plasmonic Bio-sensing. Plasmonics 2010, 5, 161-167.

20. Marinakos, S. M.; Chen, S.; Chilkoti A. Plasmonic Detection of a Model Analyte in Serum by a Gold Nanorod Sensor. Anal. Chem. 2007, 79, 5278-5283.

21. Chen, C.-D.; Cheng, S.-F.; Chau, L.-K.; Chris Wang, C. R. Sensing capability of the localized surface plasmon resonance of gold nanorods. Biosens. Bioelectron. 2007, 22, 926-932.

22. Li, C.; Wu, C.; Zheng, J.; Lai, J.; Zhang, C.; Zhao Y. LSPR Sensing of Molecular Biothiols Based on Noncoupled Gold Nanorods. Langmuir 2010, 26, 9130-9135. 
23. Yu, C.; Irudayaraj, J. Multiplex Biosensor Using Gold Nanorods. Nano Lett. 2007, 79, 572-579.

24. Mayer, K. M.; Hao, F.; Lee, S.; Nordlander, P.; H Hafner, J. H. A single molecule immunoassay by localized surface plasmon resonance. Nanotechnology 2010, 21, 255503-8.

25. Nehl, C. L.; Liao, H.; Hafner, J. H. Optical Properties of Star-Shaped Gold Nanoparticles. Nano Lett. 2006, 6, 683-688.

26. Ament, I.; Prasad, J.; Henkel, A.; Schmachtel, S.; Sonnichsen, C. Single Unlabeled Protein Detection on Individual Plasmonic Nanoparticles. Nano Lett. 2012, 12, 10921095.

27. Zijlstra, P.; Paulo, P. M. R.; Orrit, M. Optical detection of single non-absorbing molecules using the surface plasmon resonance of a gold nanorod. Nat. Nanotechnol. 2012, 7, 379-382.

28. Lu, G.; Hou, L.; Zhang, T.; Li, W.; Liu, J.; Perriat, P.; Gong, Q. Anisotropic Plasmonic Sensing of Individual or Coupled Gold Nanorods. J. Phys. Chem. C 2011, 115, 2287722885.

29. Beeram, S. R.; Zamborini, F. P. Selective Attachment of Antibodies to the Edges of Gold Nanostructures for Enhanced Localized Surface Plasmon Resonance Biosensing. J. Am. Chem. Soc. 2009, 131, 11689-11691. 
30. Beeram, S. R.; Zamborini, F. P. Effect of Protein Binding Coverage, Location, and Distance on the Localized Surface Plasmon Resonance Response of Purified Au Nanoplates Grown Directly on Surfaces. J. Phys. Chem. C 2011, 115, 7364-7371.

31. Feuz, L.; Jonsson, M. P.; Höök, F. Material-Selective Surface Chemistry for Nanoplasmonic Sensors: Optimizing Sensitivity and Controlling Binding to Local Hot Spots. Nano Lett. 2012, 12, 873-879.

32. Zijlstra, P.; Paulo, P. M. R.; Yu, K.; Xu, Q. H.; Orrit, M. Chemical Interface Damping in Single Gold Nanorods and Its Near Elimination by Tip-Specific Functionalization. Angew. Chem. Int. Ed. Engl. 2012, 51, 8352-8355.

33. Fang, A.; White, S. L.; Masitas, R. A.; Zamborini, F. P.; Jain, P. K. One-to-One Correlation between Structure and Optical Response in a Heterogeneous Distribution of Plasmonic Constructs. J. Phys. Chem. C 2015, 119, 24086-24094.

34. Cortés, E.; Xie, W.; Cambiasso, J.; Jermyn, A. S.; Sundararaman, R.; Narang, P.; Schlücker, S.; Maier, S. A. Plasmonic hot electron transport drives nano-localized chemistry. Nat. Commun. 2017, 8, 14880-10.

35. Yurkin, M. A.; Hoekstra, A. G. The Discrete-Dipole-Approximation Code ADDA: Capabilities and Known Limitations. J. Quant. Spectrosc. Radiat. Transfer 2011, 112, 2234-2247.

36. Johnson, P. B.; Christy, R. W. Optical Constants of Noble Metals. Phys. Rev. B 1972, 6, 4370-4379. 
37. D’Agostino, S.; Pompa, P. P.; Chiuri, R.; Phaneuf, R.; Britti, D. G.; Rinaldi, R.; Cingolani, R.; Della Sala, F. Enhanced Fluorescence by Metal Nanospheres on Metal Substrates. Opt. Lett. 2009, 34, 2381-2383.

38. Vasina, E. N.; Déjardin, P. Kinetics of Adsorption, Desorption, and Exchange of $\alpha-$ Chymotrypsin and Lysozyme on Poly(ethyleneterephthalate) Tracked Film and TrackEtched Membrane. Biomacromolecules 2003, 4, 304-313.

39. Snopok, B. A.; Darekarb, S.; Kashuba, E. V. Analysis of protein-protein interactions in a complex environment: capture of an analyte-receptor complex with standard additions of the receptor (CARSAR) approach. Analyst 2012, 137, 3767-3772.

40. Haussling, L.; Ringsdorf, H.; Schmitt F.-J.; Knoll, W. Biotin-Functionalized Self Assembled Monolayers on Gold: Surface Plasmon Optical Studies of Specific Recognition Reactions. Langmuir 1991, 7, 1837-1840.

41. Pérez-Luna, V. H.; O’Brien, M. J.; Opperman, K. A.; Hampton, P. D.; López, G. P.; Klum, L. A.; Stayton, P. S. Molecular Recognition between Genetically Engineered Streptavidin and Surface-Bound Biotin. J. Am. Chem. Soc. 1999, 121, 6469-6478.

42. Jung, L. S.; Nelson, K. E.; Stayton, P. S.; Campbell, C. T. Binding and dissociation kinetics of wild type and mutant streptavidins on mixed biotin-containing alkylthiolate monolayers. Langmuir 2000, 16, 9421-9432.

43. Nelson, K. E.; Gamble, L.; Jung, L. S.; Boeckl, M. S.; Naeemi, E.; Golledge, S. L.; Sasaki, T.; Castner, D. G.; Campbell, C. T.; Stayton, P. S. Surface Characterization of 
Mixed Self-Assembled Monolayers Designed for Streptavidin Immobilization. Langmuir 2001, 17, 2807-2816.

44. Spinke, J.; Liley, M.; Schmitt, F. J.; Guder, H. J.; Angermaier, L.; Knoll, W. Molecular Recognition at Self-Assembled Monolayers - Optimization of Surface Functionalization. J. Chem. Phys. 1993, 99, 7012-7019.

45. Weber, P. C.; Ohlendorf, D. H.; Wendoloski, J. J.; Salemme, F. R. Structural origins of high-affinity biotin binding to streptavidin. Science 1989, 243, 85-88.

46. Chilkoti, A.; Stayton, P. S. Molecular Origins of the Slow Streptavidin-Biotin Dissociation Kinetics. J. Am. Chem. Soc. 1995, 117, 10622-10628.

47. Cheng, S.-F.; Chau, L.-K. Colloidal Gold-Modified Optical Fiber for Chemical and Biochemical Sensing. Anal. Chem. 2003, 75, 16-21.

48. Tang, Y.; Mernaugh, R.; Zeng, X. Nonregeneration Protocol for Surface Plasmon Resonance: Study of High-Affinity Interaction with High-Density Biosensors. Anal. Chem. 2006, 78, 1841-1848.

49. Wang, Y.; Qian, W.; Tan, Y.; Ding, S. A label-free biosensor based on gold nanoshell monolayers for monitoring biomolecular interactions in diluted whole blood. Biosens. Bioelectron. 2008, 23, 1166-1170.

50. Nath, N.; Chilkoti, A. A Colorimetric Gold Nanoparticle Sensor To Interrogate Biomolecular Interactions in Real Time on a Surface. Anal. Chem. 2002, 74, 504-509. 
51. Nath, N.; Chilkoti, A. Label-Free Biosensing by Surface Plasmon Resonance of Nanoparticles on Glass: Optimization of Nanoparticle Size. Anal. Chem. 2004, 76, 5370-5378.

52. Arai, T.; Kumar, P. K. R.; Rockstuhl, C; Awazu, K.; Tominaga, J. An optical biosensor based on localized surface plasmon resonance of silver nanostructured films. J. Opt. A: Pure Appl. Opt. 2007, 9, 699-703.

53. Liao, W.-S.; Chen, X.; Yang, T.; Castellana, E. T.; Chen, J.; Cremer, P. S. Benchtop chemistry for the rapid prototyping of label-free biosensors: Transmission localized surface plasmon resonance platforms. Biointerphases 2009, 4, 80-85.

54. Haes, A. J.; Van Duyne R. P. A Nanoscale Optical Biosensor: Sensitivity and Selectivity of an Approach Based on the Localized Surface Plasmon Resonance Spectroscopy of Triangular Silver Nanoparticles. J. Am. Chem. Soc. 2002, 124, 1059610604.

55. Mayer, K. M.; Lee, S.; Liao, H.; Rostro, B. C.; Fuentes, A.; Scully, P. T.; Nehl, C. L.; Hafner, J. H. A Label-Free Immunoassay Based Upon Localized Surface Plasmon Resonance of Gold Nanorods. ACS Nano 2008, 2, 687-692.

56. Nusz, G. J.; Curry, A. C.; Marinakos, S. M.; Wax, A.; Chilkoti, A. Rational Selection of Gold Nanorod Geometry for Label-Free Plasmonic Biosensors. ACS Nano 2009, 3, 795-806. 


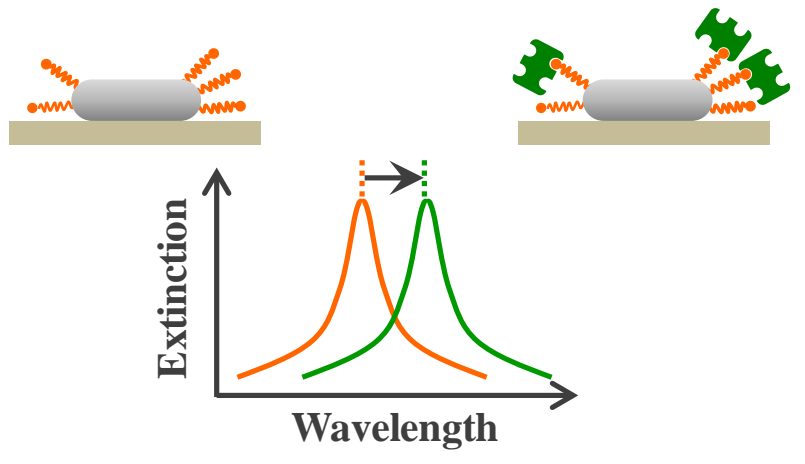

Table of Contents Graphic (TOC) 